\section{$I 5-433$}

Electrical Properties of the Tungsten Bronzes*

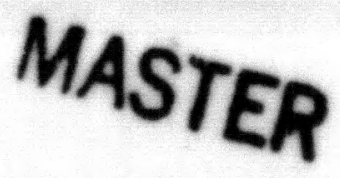

H. R. SHANKS, P. I1. SIDLES, AND G. C. DANIELSON

Institute for Atomic Research and Department of Physics

lowa State University, Ames, lowa

Abstract-The electrical resistivity of $\mathrm{Na}_{x} \mathrm{WO}_{3}, \mathrm{Li}_{x} \mathrm{WO}_{3}$, and $\mathrm{K}_{\mathrm{x}} \mathrm{WO}_{3}$ has been measured at $300^{\circ} \mathrm{K}$. The range of $\mathrm{x}$-values was $0.25<x<0.9$. All resistivities were characteristic of a metal and lie on a single curve. An extrapolation of the conductivity curve to zero conductivity indicated that the tungsten bronzes should be semiconductors for $x<0,25$. The resistivities that have been measured for tungsten bronzes with $x<0.25$ showed semiconducting behavior. The resistivity of $\mathrm{Li}_{\mathbf{x}} \mathrm{WO}_{3}$ exhibited an anomalous peak in the $p \mathrm{vs} \mathrm{T}$ curve. The Hall coefficient of $\mathrm{Li}_{0}, 37 \mathrm{WO}_{3}$ indicated one free electron per alkali atom as was previously found for $\mathrm{Na}_{\mathbf{x}} \mathrm{WO}_{3}$. The Seebeck coefficient of $\mathrm{Na}_{x} \mathrm{WO}_{3}$ depended linearly on $x^{-2 / 3}$ as expected from free electron theory. The implications of these and some other data are discussed.

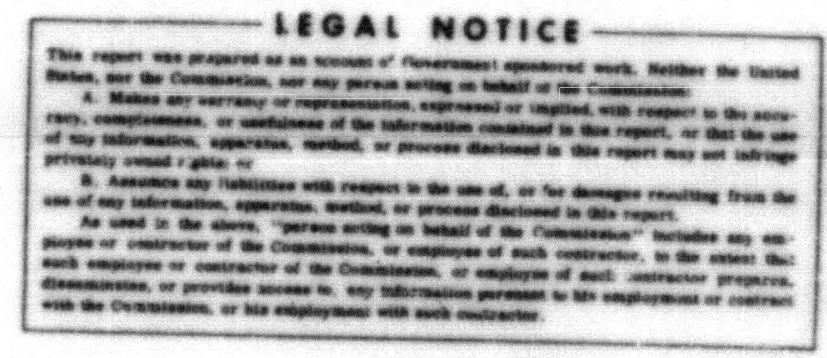




\section{INTRODUCTION}

Tungsten bronzes are non-stiochiometric compounds $\mathrm{M}_{\mathrm{x}} \mathrm{wO}_{3}$ where M is usually one of the alkali metals. Single crystals of these compounds, large enough for electrical measurements, can be prepared with values of $x$ ranging from essentially zero to nearly unity. The tungsten bronzes undergo several changes of crystal structure as $x$ changes, but difficulties in determining quantitative amounts of $M$ have frustrated attempts to delineate the ranges in $x$ over which each of these structures exists. This situation has been a deterrent to studies of the electrical properties of these materials, especially in the low $x$-value range.

Electrical properties of certain of the high $\mathrm{x}$-value bronzes have been previously reported. Brown and Banks ${ }^{1}$ and Gardner and Danielson ${ }^{2}$ both measured the electrical resistivity of cubic sodium tungsten bronze, with $x$-values ranging from about 0.5 to 0.9 , and reported a minimum in resistivity near $x=0.75$. Gardner and Danielson ${ }^{2}$ also reported the results of Hall coefficient measurements which indicated that, in this range of $x$ values, each sodium atom contributed one electron to conduction processes. Subsequentiy, Ellerbeck, et al. ${ }^{3}$ reported that, when careful attention was given to sample homogeneity, no minimum in electrical resistivity at $x=0.75$ was observed.

Alkali metal bronzes of lower $\mathrm{x}$-value have not been so extensively studied. With the exception of some measurements by Sienke and Truong ${ }^{4}$ 
of the electrical conductivity of cubic lithium bronzes, little of significance has been reported. It is the purpose of this publication to report the results of electrical resistivity measurements on several metal-1ike tungsten bronzes, with $\mathrm{x}$-values down to 0.28 , together with some preliminary results for Hall and Seebeck coefficients, and to discuss the implications of these results. Below about $x=0.25$, all of the alkali tungsten bronzes appear to exhibit properties which are characteristic of semiconductors. Some preliminary results are shown which illustrate this type of behavior.

\section{CRYSTAL PREPARATION}

Crystals of the various alkali tungsten bronzes were prepared by electrolysis from a melt of the appropriate alkali tungstate and $\mathrm{wO}_{3}$. The electrolytic cell consisted of a glazed ceramic crucible, a chromel wire cathode, and a graphite anode. Crystals were obtained under the following conditions: temperature of the inelt, $750-900^{\circ} \mathrm{C}$; current through the cell, 15-50 ma; time of electrolysis, 12-24 hr.

For the low $x$-value sodium tungsten bronzes $(x<0.5)$, it was found that the crystal structure and $x$-values of the crystals obtained depended strungly on the temperature of the melt. The size and homogeneity of the crystals were dependent on both temperature and electrode current. The best crystals were obtained at the lowest temperature at which they could be grown. The optimum current for best quality crystals depended upon $x$-value and erystal structure. 


\section{MEASUREMENTS}

Measurements of electrical resistivity were carried out by a dc method using 4-probe techniques to avoid problems arising from contact resistance. Pressure contacts were used for both current and potential probes. At low temperatures, the current contacts could be improved by ultrasonically tinning the ends of the samples.

Details of the method employed for measuring Seebeck coefficients have been previously described by Heller and Danielson. ${ }^{5}$ The Hall coefficient of $\mathrm{Li}_{0.37}{ }_{3} \mathrm{WO}_{3}$ was measured by a dc method and is therefore subject to error from the Ettingshousen effect. This arroz ta not expected to exceed $\pm 10 \%$.

\section{ELECTRICAL RESISTIVITY}

In $\mathbf{F}$ g. 1, the resistivities of several tungsten bronzes at $300^{\circ} \mathrm{K}$ are shown as a function of the alkali metal concentrations. Experimental points are shown for cubic $\mathrm{Na}_{\mathbf{x}} \mathrm{WO}_{3}$, tetragonal $\mathrm{Na}_{\mathbf{x}} \mathrm{wO}_{3}$, cubic $\mathrm{Li}_{\mathbf{x}} \mathrm{wO}_{3}$ (including data both from Iowa State University and Cornell University ${ }^{4}$ ). and tetragonal $\mathrm{K}_{\mathbf{x}} \mathrm{WO}_{3}$. All of these bronzes show metallic conductivity. It is remarkable that the resistivities for all these different bronzes seem to fall on the same curve. The metal ions themselves cannot, therefore, be important contributors to the scattering of the free electrons. Rather, the mobility must be limited primarily by electron scattering from the acoustical and optical modes of the $\mathrm{WO}_{3}$ structure at high temperafures, 
and from the vacancies at alkali metal sites at low temperatures. The importance of vacancy scattering at low temperatures has been shown by Ellerbeck et al. ${ }^{3}$ The conductivity at $0^{\circ} \mathrm{K}$ (see their Fig. 4) increased rapidly with increasing sodium concentration owing to a reduction in the number of vacancies which scatter electrons as $\mathbf{x}$ increased.

In Fig. 2, the conductivities of these same bronzes at $300^{\circ} \mathrm{K}$ are plotted $v s x$. These conductivities are simply the reciprocals of the resistivities shown in Fig. 1. By extrapolation to zero conductivity, the curve in Fig. 2 strongly suggests that all the different bronzes will become insulators (or semiconductors) for values of $x$ less than some value which is in the neighborhood of 0.25 . This possibility has been suggested by Sienko and Truong ${ }^{4}$ who used the theory of Mott $^{6}$ for metal-semiconducter transitions. The experimental data did not, however, show evidence for the discontinuity that would be expected from Mott's theory. If small, the discontinuity may be very difficult to observe experimentally owing to insufficient homogeneity of most available crystals. We have grown a number of tungsten bronze crystals with $x<0.25$, and have found that such crystals were semiconductors. No crystals with $x<0.25$ have been found to be metallic in conductivity.

The electrical resistivity of one of these semiconducting bronzes is shown in Fig. 3. The crystal is $\mathrm{Li}_{\mathbf{x}} \mathrm{WO}_{3}$ with $\mathrm{x}=0.097$. The graph in Fig. 3 of $\log p$ vs $100 / \mathrm{T}$ shows the typical behavior of an impurity semi- 
conductor. The activation energy corresponding to the straight line at low temperatures (extrinsic region) was $0.03 \mathrm{ev}$, the activation energy corresponding to the straight line at high temperatures was $0.12 \mathrm{ev}$. If the straight line at high temperatures corresponded to the intrinsic region, the energy gap for this semiconducting crystal was about 0.24 ev.

The temperature dependence of the resistivity of $\mathrm{Li}_{\mathrm{x}} \mathrm{WO}_{3}$ is shown in Fig. 4. For $x=0.28$, the anomalous peak was very large and oceurred at about $600^{\circ} \mathrm{K}$; for $\mathrm{x}=0,34$ the peak was much smaller and occurred at about $300^{\circ} \mathrm{K}$. With increasing lithium concentration, the peak, therefore, diminished in size and shifted to lower temperatures. The peak was completely reproducible and $x$ ray diffraction patterns showed that the cubic crystal structure existed both below and above the temperature at which the peak occurred. However, preliminary thermal analysis measurements indicated some sort of phase change. Mackintosh ${ }^{7}$ has suggested the possibility of ordering of the lithium atoms, and neutron diffraction studies of these cubic $\mathrm{Li}_{\mathbf{x}} \mathrm{WO}_{3}$ crystals should be made below and above the transition temperature.

\section{HALL EFFECT}

The Hall coefficient ( $R$ ) of a crystal of cubic $\mathrm{Li}_{0.37} \mathrm{WO}_{3}$ has been measured at $300^{\circ} \mathrm{K}$. From this measurement, the number of free electrons per unit volume (n) was calculated from $n=1 /$ Re, where $e$ is the charge on one electron. Hence the numbe $t$ of free electrons per mole was obtained. 
This result for $\mathrm{Li}_{0}, 37 \mathrm{WO}_{3}$ is shown in Fig. 5, along with similar results for $\mathrm{Na}_{x}$ wO $\mathrm{O}_{3}$ obtained by Gardner and Danielson. ${ }^{2}$ The straight line corresponds to one free electron per alkali metal atom, and the fact that the point for $\mathrm{Li}_{0},{ }_{37} \mathrm{WO}_{3}$ is very near this line strongly suggests that the number of free electrons in $\mathrm{Li}_{\mathbf{x}} \mathrm{WO}_{3}$, as in $\mathrm{Na}_{\mathbf{x}} \mathrm{WO}_{3}$, is equal to the number of alkali metal atoms.

\section{SEEBECK EFFECT}

The Seebeck coefficients (thermoelectric powers) of $\mathrm{Na}_{\mathbf{x}} \mathrm{WO}_{3}$ have been measured over a wide range of $x$-values at room temperature $\left(300^{\circ} \mathrm{K}\right)$. At this temperature, the residual resistance $\left(\rho_{0}\right)$ and thermal resistance $\left(p_{t}\right)$ are comparable, the value of $p_{0}$ being between $p_{t}$ and $2 p_{t}$. Nevertheless, one would expect to a first approximation ${ }^{8}$ that $S=(1 / 3)\left(\pi^{2} k^{2} T / e \xi\right)$. where $S$ is the Seebeck coefficient, $k$ is Boltzmann's constant, e is the electronic charge, and $\boldsymbol{b}$ is the Fermi energy. For free electrons, the Fermi energy $\zeta=\left(h^{2} / 2 m^{*}\right)(3 n / 8 \pi)^{2 / 3}$ where $h$ is Planck's constant, $m^{*}$ is the effective mass, and $\mathrm{n}$ is the density of free electrons. Since $\mathrm{n}$ is proportional to $x, \zeta$ varies as $x^{2 / 3}$ and $S$ varies as $x^{-2 / 3}$.

In Fig. 6, the Seebeck coefficient (S) is plotted vs $x^{-2 / 3}$. The experimental points lie on a straight line which provides evidence for the validity of free electron theory when discussing transport properties of the tungsten bronzes. From the slope of the straight line, the ratio of the effective mass to the true mass of the electron $\left(\mathrm{m}^{*} / \mathrm{m}\right)$ was calculated 
and found to have the eurprisingly small value of about 0.035 .

In Fig. 7, the Seebeck coefficient (S) is plotted vs the absolute temperature ( $T$ ). At low temoeratures, where the residual resistance dominated the thermal resistance, the expression $S=(1 / 3)\left(\pi^{2} k^{2} T / e \zeta\right)$ should be valid if free electron theory is applicsble. We do indeed find in Fig. 6 a linear dependence of $\mathrm{S}$ upon $\mathrm{T}$ at low temperatures as expected. From the slope of the straight line at low temperatures we calculated $\mathrm{m}^{*} / \mathrm{m}$, and again found a small value of about 0.037 .

\section{DISCUSSION}

The transport properties of the tungsten bronzes appear to agree with the properties which would be expected from a free electron model. In particular, the thermal part of the electron mobility is nearly independent of the $\mathrm{x}$-value; the Hall coefficient gives the correct number of charge carriers; and the Seebeck coefficient varies linearly with $x^{-2 / 3}$. These electrical properties suggest that the density of states in the conduction band $g(\varepsilon)$ is given in terms of the energy $(\varepsilon)$ according to the free electron model $g(\varepsilon) d \varepsilon=\varepsilon^{1 / 2} \mathrm{~d} c$. The Seebeck data also indicate a small effective mass. Since the mobility $\mu=\left(\mathrm{e} / \mathrm{m}^{*}\right) \tau$, the low value for $\mathrm{m}^{*}$ implies a very short relaxation time (T). For example, at $x=0.7, \mu \sim 20 \mathrm{~cm}^{2} / \mathrm{v}-\mathrm{sec}$; and $m^{*}=0.035$ gives a relaxation time $\tau=4 \times 10^{-15} \mathrm{sec}$.

On the other hand, the thermodynamic properties of the bronzes, suggest that the density of states varies much more rapidly with energy than 
$e^{1 / 2}$. Magnetic susceptibility data by Greiner, Shanks, and Wallace ${ }^{9}$ as well as specific heat data by Vest, Griffel, and Smith ${ }^{10}$ definitely show that $g(\varepsilon) d \varepsilon=\varepsilon^{1 / 2} \mathrm{~d} \varepsilon$ is not valid; but botk sets of data (if we ignore the specific heat data for large values of $x$ ) indicate an energy degendence of the density of states which is the same and considerably greater than $e^{1 / 2}$. Furthermore, the best fit using $e^{1 / 2}$ gives $\mathrm{m}^{*} / \mathrm{m}=1.6$, which ts far greater than the value 0.035 given by Seebeck measurements. This discrepancy suggests that free electron theory must be considerably modified to account for the thermodynamic properties of the bronzes, however vell this theory explains some of the transport properties. 


\section{REFER ENCES}

*Contribution No. 1133. Work was performed in the Ames Laboratory of the U. S. Atomic Energy Commission.

${ }^{1}$ B. W. Brown and E. Banks, Phys. Rev. 84, 609 (1951).

${ }^{2}$ W. R. Gardner and G. C. Danielson, Phys. Rev. 93, 46 (1954).

${ }^{3}$ L. D. Ellerbeck, H. R. Shanks, P. H. Sidles, and G. C. Danielson, J. Chem. Phys. 35, 298 (1961).

${ }^{4}$ M. J. Sienko and Thu Ba Nguyen Truong, J. Am. Chem. Soc. 8s, 3939 (1961).

${ }^{5}$ M. W. Heller and G. C. Danielson, J. Phys. Chem. Solids (in press).

${ }^{6}$ N. F. Mott, Nuovo Cimento ?, 312 (1958); Phil. Mag. 6, 287 (1961).

${ }^{7}$ A. R. Mackintosh, private communication.

${ }^{8}$ A. H. Wilson, Theory of Metals (Cambridge University Press, 1954), p. 286 .

9. D. Greiner, H. R. Shanks, D. C. Wallace, J. Chem. Phys. (in press).

10 R. W. Vest, M. Griffel, and J. F. Smith, J. Chem. Phys. 28, 293 (1958). 


\section{LIST OF CAPTIONS}

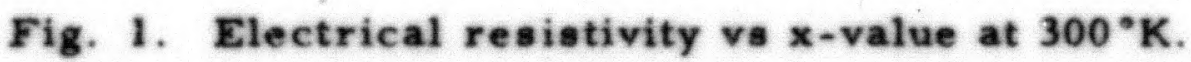

Fig. 2. Electrical conductivity vs $\mathrm{x}$-value at $300^{\circ} \mathrm{K}$.

Fig. 3. Electrical resistivity vs temperature for a semiconducting lithium bronze.

Fig. 4. Electrical resistivity vs temperature for some metallic lithium bronzes.

Fig. 5. Electren concentration vs $x$-value.

Fig. 6. Seebeck coefficient vs $x^{-2 / 3}$ for cubic sodium bronze.

Fig. 7. Seebeck coefficient vs temperature for a cubic sodium bronze. 


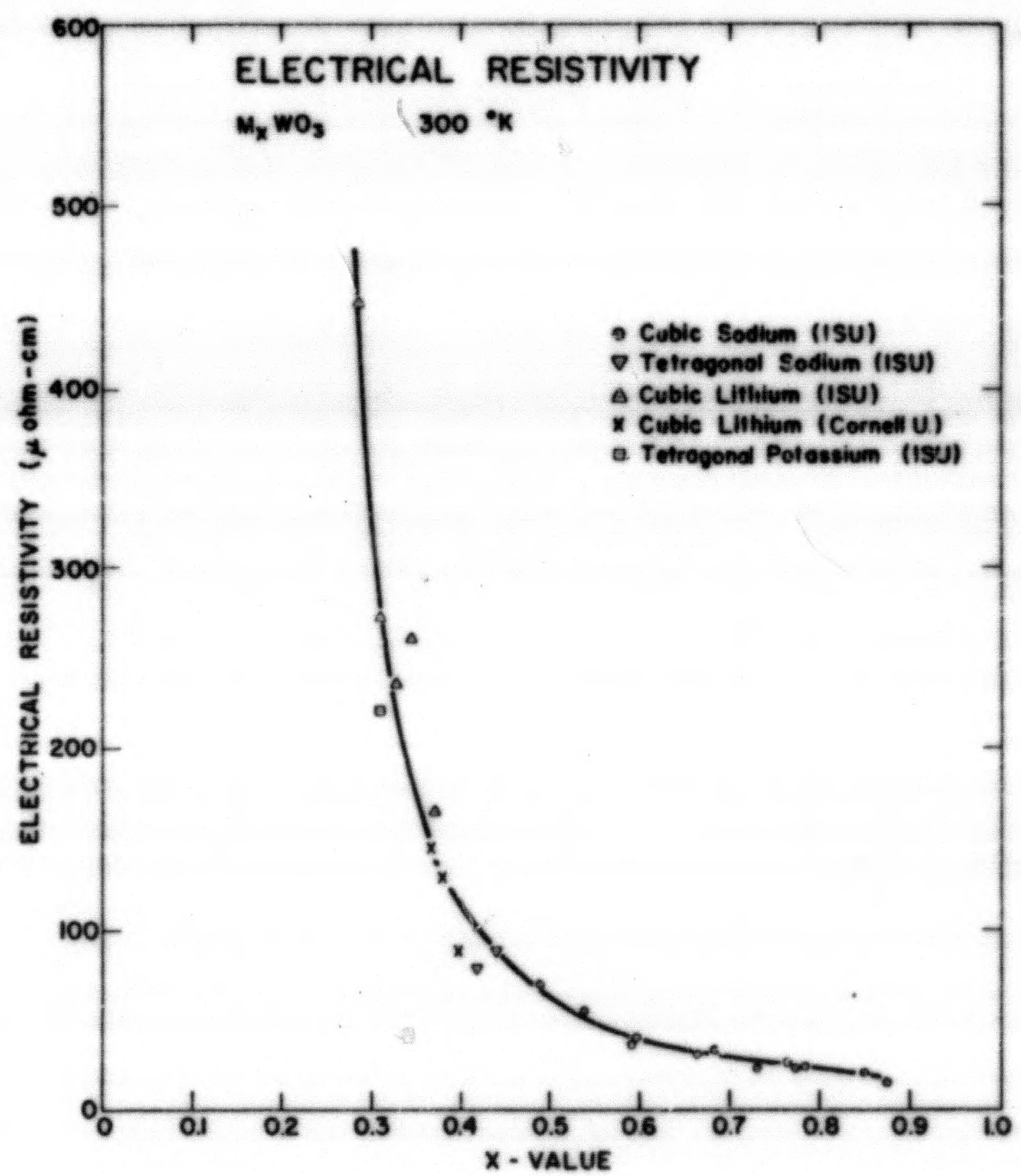

Fig. 1. Electrical resistivity vs $\mathrm{x}$-value at $300^{\circ} \mathrm{K}$ 


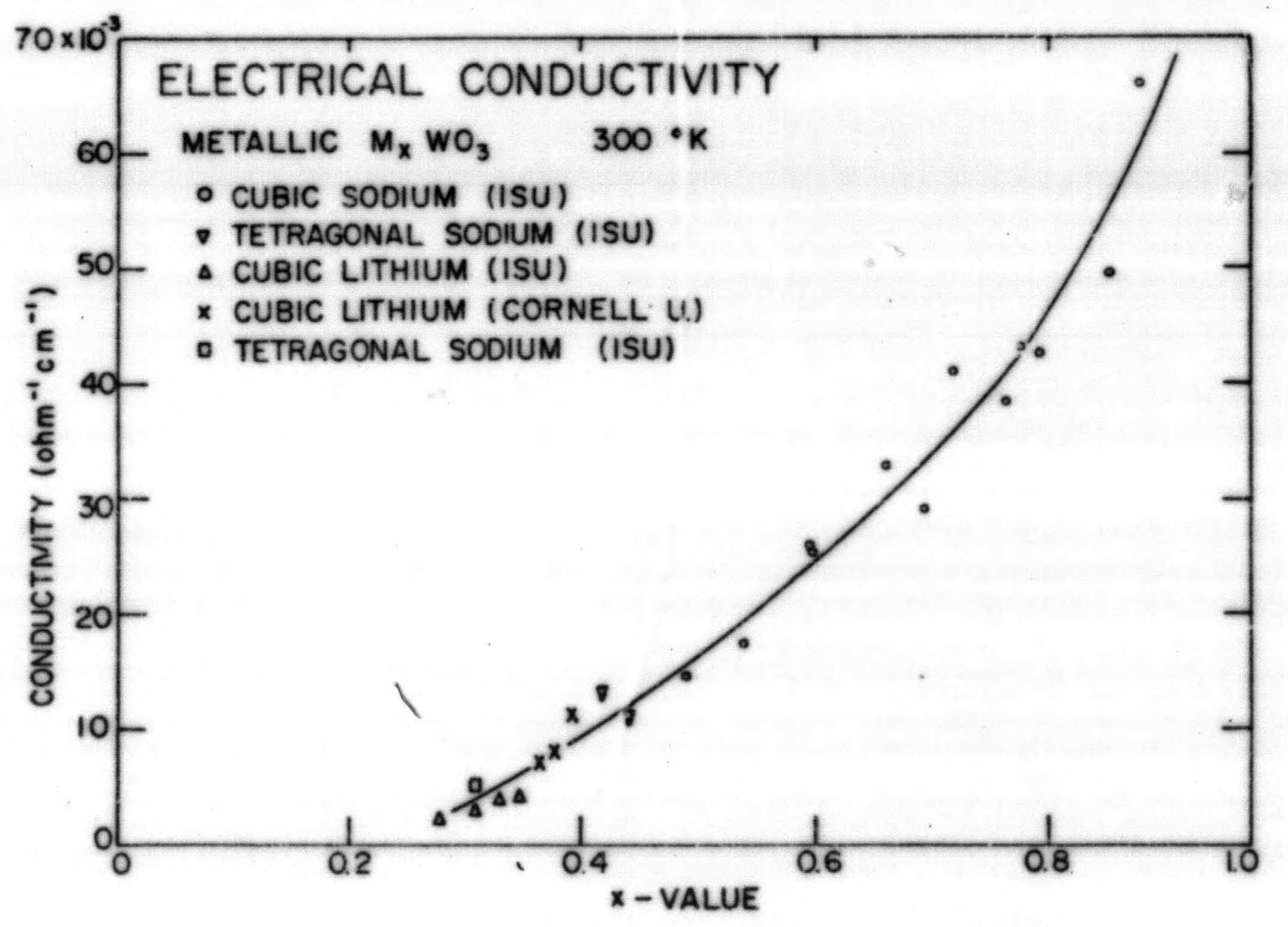

Fig. 2. Electrical conductivity vs $x$-value at $300^{\circ} \mathrm{K}$. 


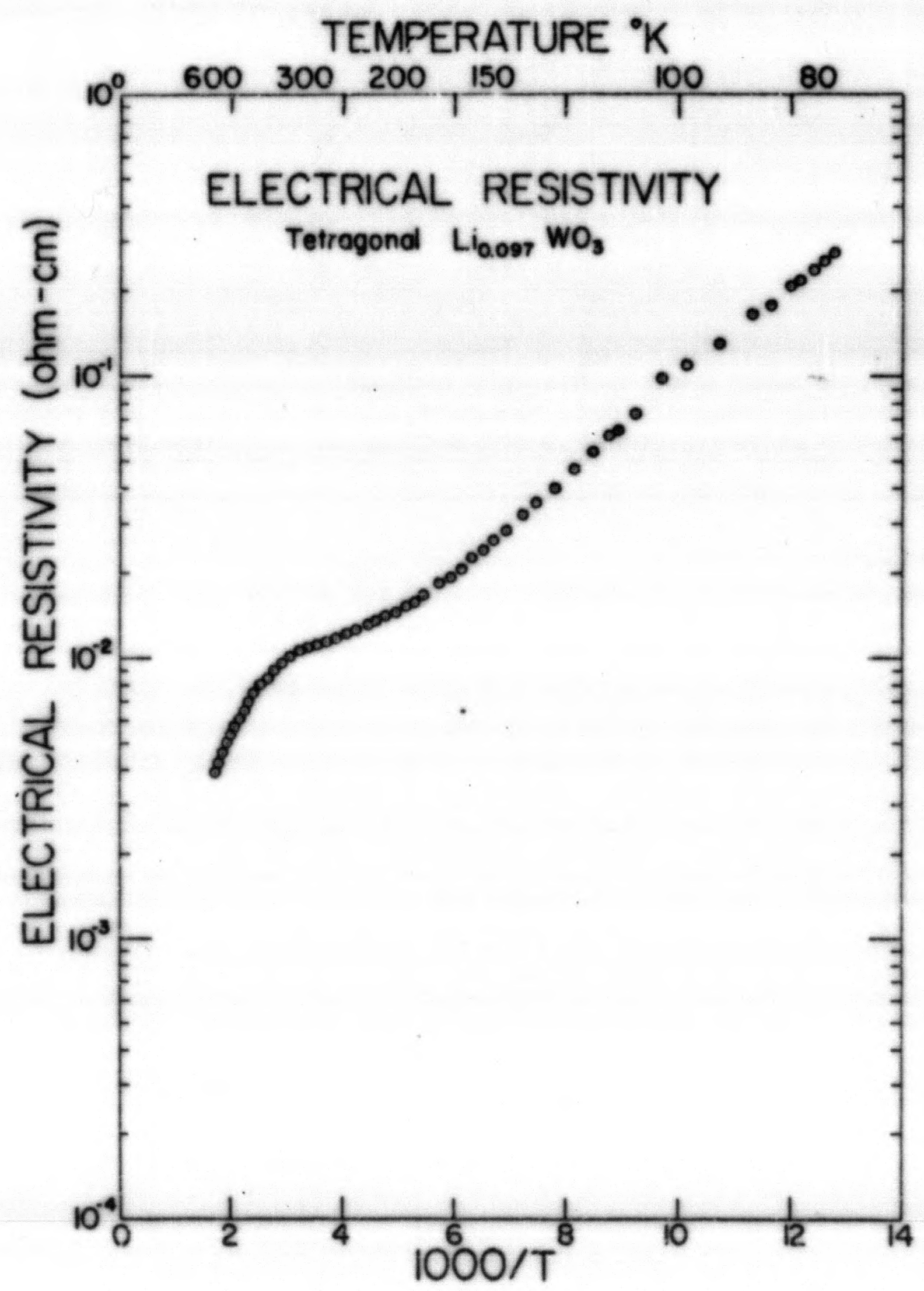

Fig. 3. Electrical resistivity vs temperature for a semiconducting lithium bronze. 


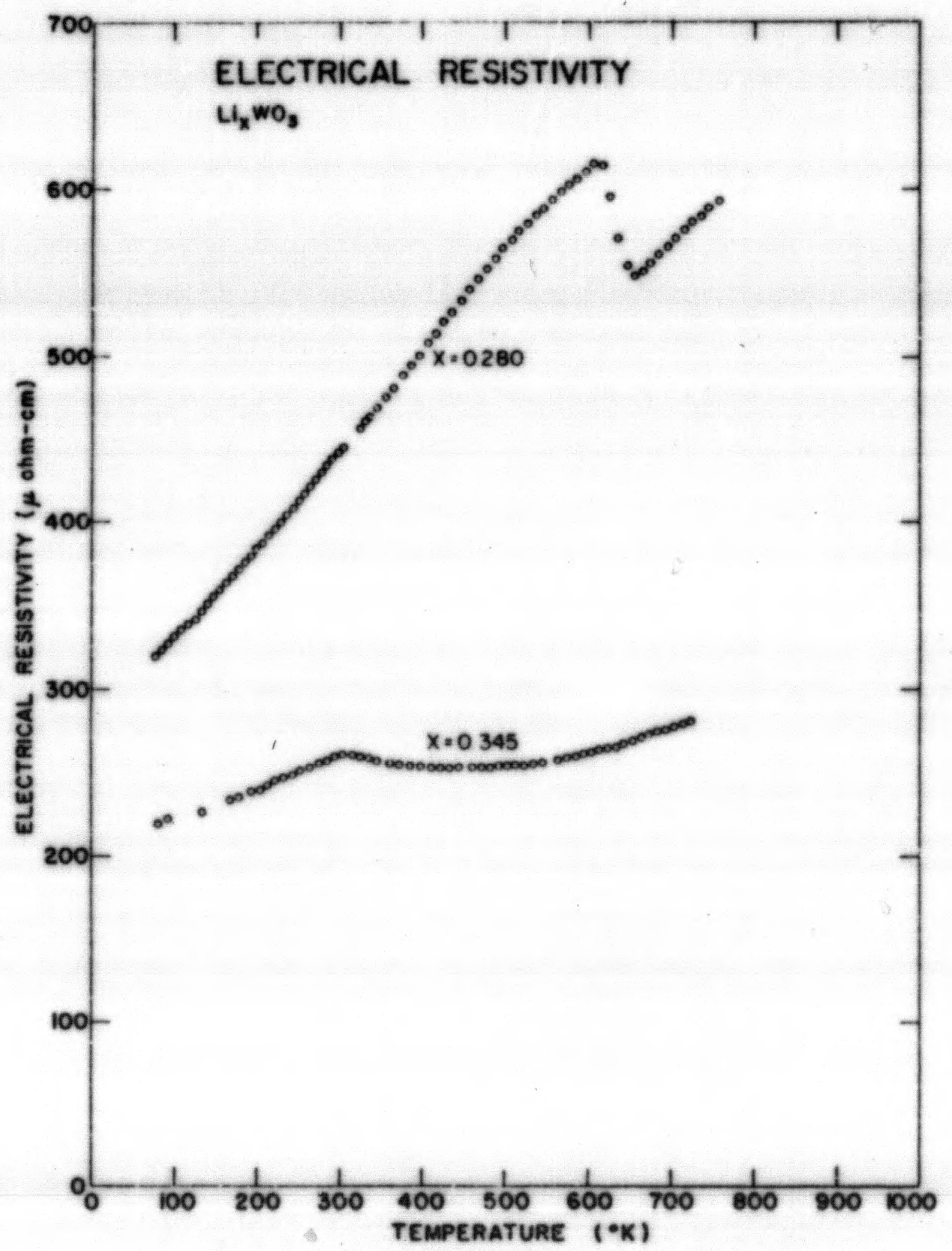

Fig, 4. Electrical resistivity vs temperature for some metallic lithium bronzes. 


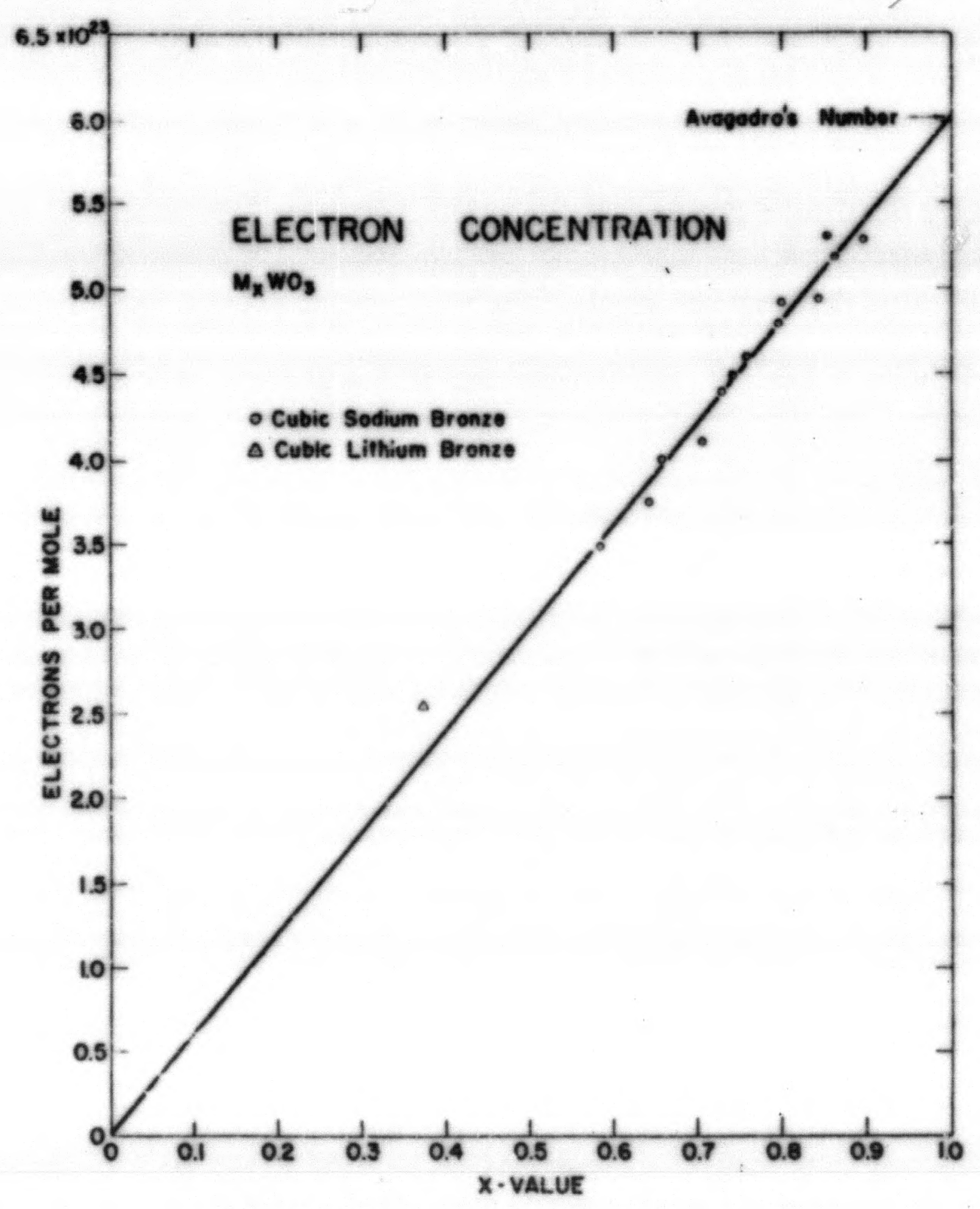

Fig. 5. Electron concentration vs x-value. 


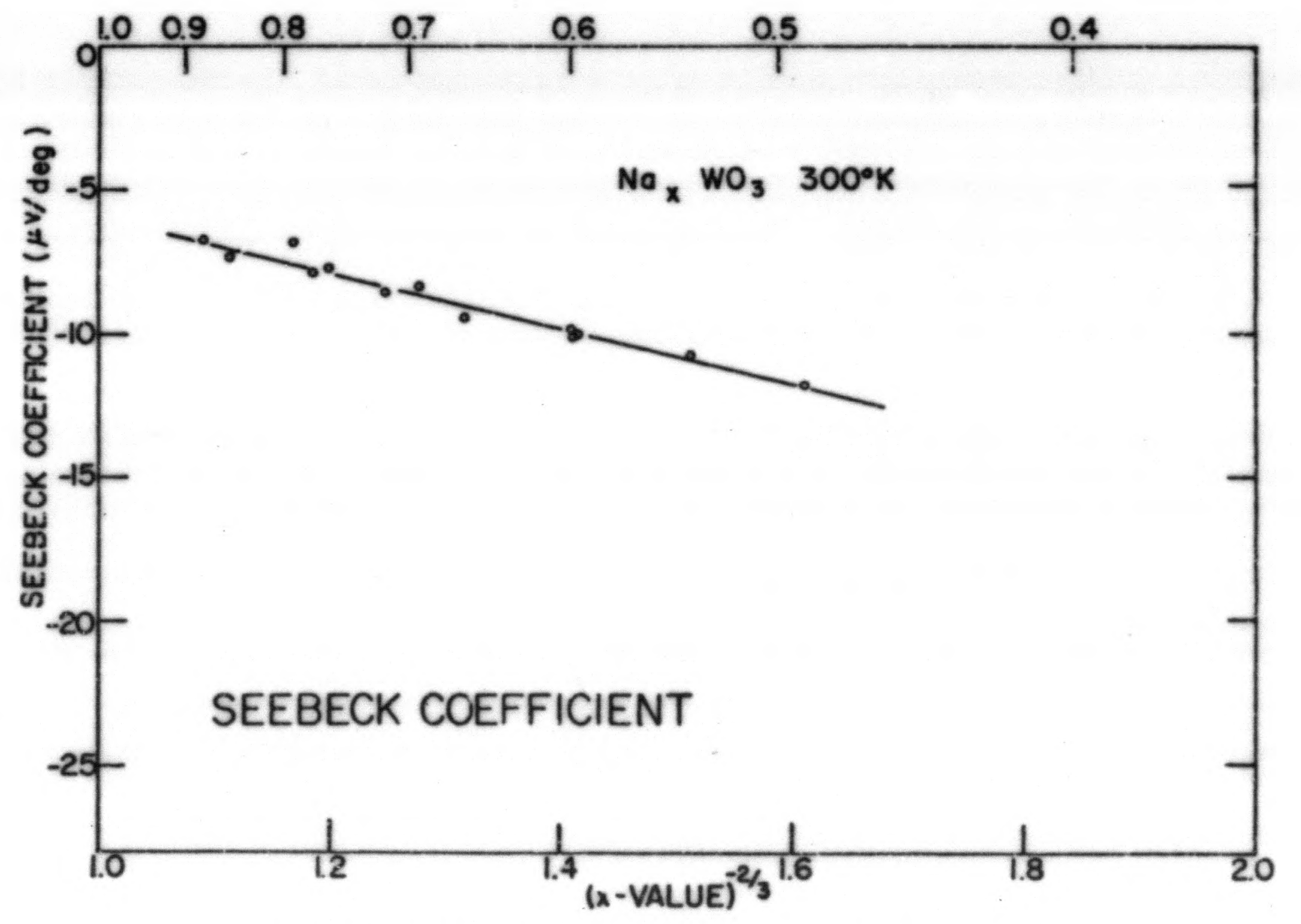

Fig. 6. Seebeck coefficient $v s \mathrm{x}^{-23}$ for cubic sodium bronze. 


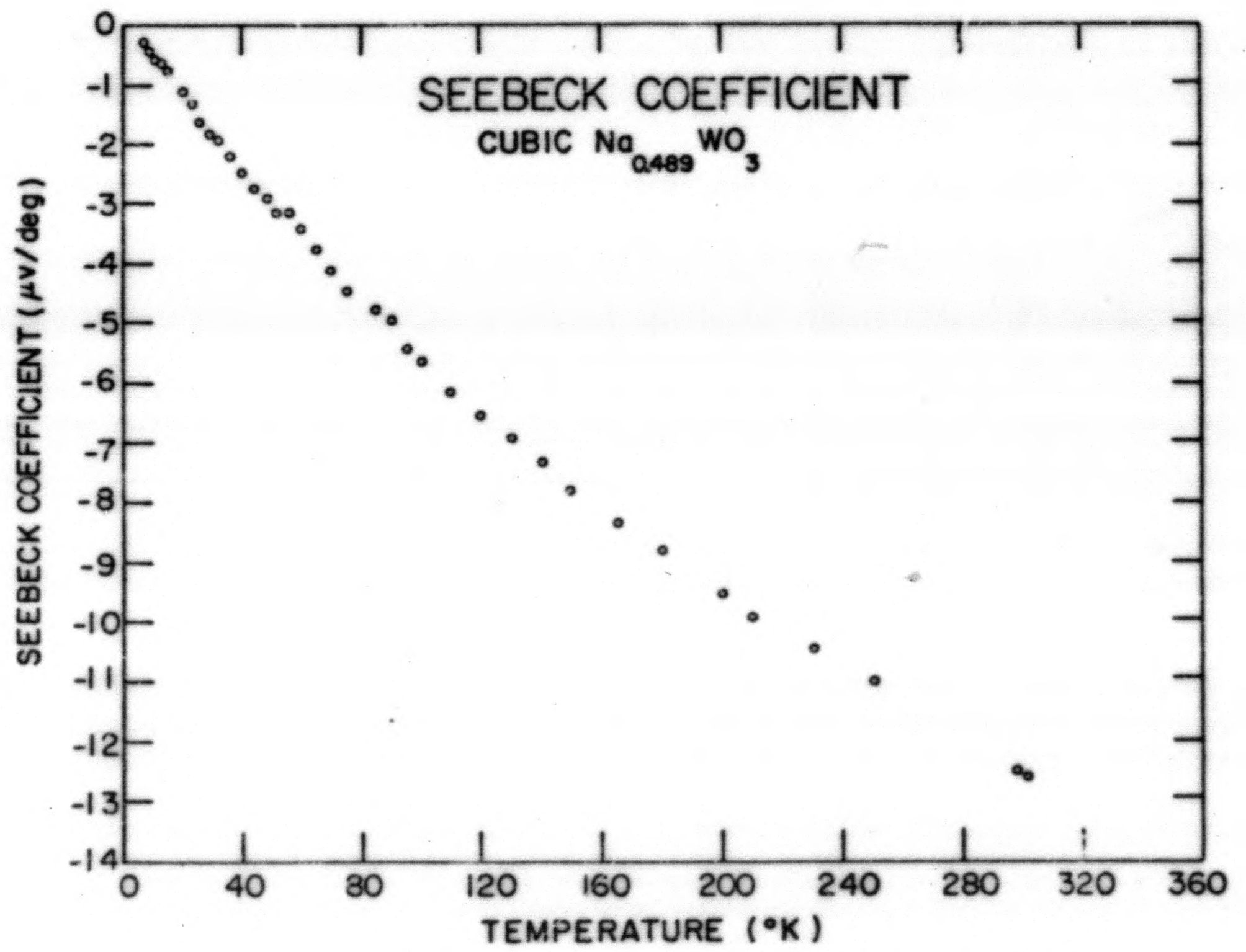

Fig. 7. Seebeck coefficient vs temperature for a cubic sodium bronze.

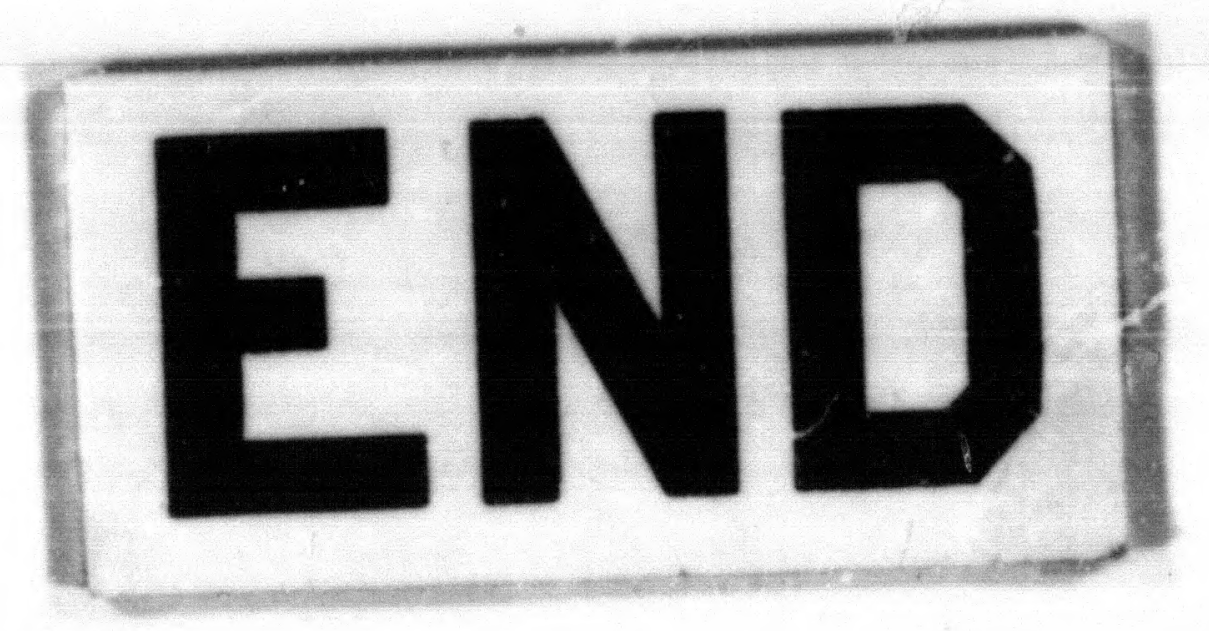

